\title{
Disturbed regulation of the isolated middle cerebral artery in acute hyponatremia
}

\author{
Marta Aleksandrowicz ${ }^{1}$, Ewa Koźniewska ${ }^{1,2}$ \\ ${ }^{1}$ Laboratory of Experimental Neurosurgery, Department of Neurosurgery, M. Mossakowski Medical Research Centre, Polish Academy \\ of Sciences, Warsaw, ${ }^{2}$ Department of Experimental and Clinical Physiology, Warsaw Medical University, Warsaw, Poland
}

\begin{abstract}
Hyponatremia is a common disorder of water-electrolyte balance characterized by the decrease of plasma sodium ions concentration below $135 \mathrm{mM}$. Although water-electrolyte balance is regulated by a kidney, symptoms of hyponatremia are related to the disturbances of intracranial homeostasis and are attributed to brain swelling. Despite the importance of blood vessels function for the homeostasis of the brain, little is known about the influence of hyponatremia on cerebrovascular regulation. In the present study isolated, perfused and pressurized rat middle cerebral arteries (MCAs) were subjected to hyponatremia in the organ chamber by lowering the concentration of sodium ions from 145 to $121 \mathrm{mM}$. The response of the MCAs to the changes in intravascular pressure and to endothelium-dependent and independent vasodilators was compared in normo- and hyponatremia. The following results were obtained: the contraction of the MCA during progressive increases in intravascular pressure was similar in normo- and hyponatremia; the dilation in response to acetylcholine $\left(10^{-6} \mathrm{M}, 10^{-5} \mathrm{M}\right.$ and $\left.10^{-4} \mathrm{M}\right)$, which is endothelium- and nitric oxide-dependent, was severely impaired in hyponatremia; the contraction after administration of the nonselective inhibitor of nitric oxide synthesis - NG-nitro-L-arginine methyl ester (L-NAME, $\left.10^{-5} \mathrm{M}\right)$ was similar in normo- and hyponatremia; the nitric oxide donor-S-nitroso- $\mathrm{N}$-acetylpenicillamine (SNAP, $10^{-5} \mathrm{M}$ ) dilated MCA only in normonatremia. The results of this study show that myogenic response and shear stress-dependent relaxation of the MCA are not affected by acute hyponatremia. However, agonist-stimulated, endothelial nitric oxide synthase (eNOS)-mediated relaxation is severely impaired which is associated with a decreased smooth muscle response to nitric oxide (NO). In conclusion, our results demonstrate that the regulation of isolated middle cerebral artery is impaired in acute hyponatremia which may contribute to the disturbance of intracranial homeostasis associated with hyponatremia.
\end{abstract}

Key words: isolated middle cerebral artery, hyponatremia, myogenic tone, acetylcholine, L-NAME, SNAP.

\section{Introduction}

Hyponatremia - the most common disturbance of water-electrolyte balance encountered in clinical practice - is defined as the lowering of sodium ion concentration in the plasma below $135 \mathrm{mM}$. Such disorder is observed in a variety of clinical conditions such as neurological diseases, heart failure, liver cirrhosis, pneumonia, tuberculosis or lung cancer [32]. In the majority of these cases, hyponatremia is related either to the syndrome of inappropriate antidiuretic hormone secretion (SIADH) or cerebral salt wasting (CSW) and is associated with hypotonicity $[2,21]$. Another cause of hyponatremia in hospital

Communicating author: Ewa Koźniewska, PhD, Laboratory of Experimental Neurosurgery, Department of Neurosurgery, M. Mossakowski Medical Research Centre, Polish Academy of Sciences, Pawińskiego 5 Str., 02-106 Warsaw, Poland, phone: +48 2260864 89, fax: +48 2266855 32, e-mail: ekozniewska@imdik.pan.pl 
settings is the therapy with loop-diuretics like e.g furosemide used to combat brain oedema in neurosurgical patients [26,27].

Hyponatremia may also develop in otherwise healthy subjects as a result of water intoxication. A typical example of such dysregulation of body fluid homeostasis is the exercise-associated hyponatremia observed in marathon runners [25]. Recently, severe symptomatic hyponatremia due to the excessive ingestion of water developing within few hours after long-lasting exercise has been reported [28].

Reduction in the extracellular concentration of $\mathrm{Na}^{+}$generates an osmotic gradient across the cell membrane and leads to the inward movement of water and cellular swelling. The cells defend themselves against osmotic swelling by activation of the regulatory volume decrease (RVD) [24]. Activation of RVD starts immediately after the cell swells. The first line of defence is the extrusion of intracellular solutes together with osmotically obligated water. The osmolytes being extruded at the onset of RVD are the main intracellular ions $\mathrm{K}^{+}$and $\mathrm{Cl}^{-}$. $\mathrm{K}^{+}$ions leave the cell through specific potassium channels such as voltage-dependent $(\mathrm{Kv})$, large conductance calciumdependent $\left(\mathrm{BK}_{\mathrm{Ca}}\right)$, stretch-activated $\mathrm{K}^{+}$channels [22,23].

Although water-electrolyte balance is regulated by kidneys, symptoms of hyponatremia are considered to be related to disturbances of intracranial homeostasis connected with brain swelling [8]. Neurological symptoms of the disease are typically manifested when plasma sodium concentration decreases below $125 \mathrm{mM}$ and are related to the rate of its onset. Decrease in plasma sodium concentration within less than 48 hours (acute hyponatremia) can result in irreversible neurologic damage, brain herniation and even death depending on the underlying neurological status. When plasma sodium concentration falls slowly (chronic hyponatremia), brain cells adapt to hypoosmolarity thanks to RVD which limits brain oedema [24].

The osmotic swelling and RVD occur in all cell types in the brain including endothelial and smooth muscle cells of the vascular wall. Whether swelling and subsequent extrusion of ions affect the function of blood vessels is largely unknown. So far, the impact of hyposmolality and/or lowering the extracellular concentration of sodium on isolated smooth muscle cells, isolated endothelial cells or on intact isolated cerebral blood vessels without endothelium has been studied mainly with reference to myogenic tone $[22,36]$. It has been demonstrated that endothelium-denuded rat cerebral arteries constrict in response to hypoosmotic challenge due to depolarization of VSMC and activation of voltage-operated $\mathrm{Ca}^{2+}$ channels [36]. On the other hand, it has been shown that when endothelial cells are subjected to hypoosmotic stress they release NO [14] which is a strong vasodilator. The effect of hyponatremia on the function of the structure composed of VSMC and endothelium, i.e. intact artery is difficult to predict.

In addition, it has been found that hypoosmotic challenge inhibits inward rectifying $\mathrm{K}^{+}$channels in isolated cerebral arterial smooth muscle cells [38]. These channels are essential for the regulation of vascular wall tension [16].

Taking into consideration that 1) cerebral blood vessels which contribute essentially to intracranial homeostasis, are also subjected to hypoosmotic challenge, 2) some of $\mathrm{K}^{+}$channels activated during RVD are essential for the regulation of cerebral circulation, 3) very little is known about the effect of hyponatremia on cerebral blood vessels function, we designed the present study to exploit the effect of acute, moderate hyponatremia on the myogenic, endothelium-dependent and endothelium-independent regulation of the isolated middle cerebral artery of the rat.

\section{Material and methods}

Male Wistar rats (body weight 280-310 g, $n=45$ animals) supplied by the Animal House of Mossakowski Medical Research Centre, Warsaw, Poland, were used in this study. Animal procedures were performed in accordance with institutional guidelines and were approved by the IV Local Commission for the Care and Use of Laboratory Animals for Experimental Procedures, National Medicines Institute in Warsaw.

\section{Isolation and mounting of the middle cerebral artery}

Rats were deeply anaesthetized with $5 \%$ isoflurane in $70 \% \mathrm{NO}_{2} / 30 \% \mathrm{O}_{2}$ and decapitated. The brain was removed from the cranium and placed in a cold $\left(4^{\circ} \mathrm{C}, \mathrm{pH}=7.4\right)$ saline solution (composition is provided at the end of this section) buffered with MOPS (3-(N-morpholino)propanesulfonic acid) containing $1 \%$ dialysed bovine serum albumin (BSA). Approximately $5 \mathrm{~mm}$ length MCA without branches was isolated starting from the circle of Willis. Parenchy- 
mal tissue was carefully removed and the vessel was transferred to an organ chamber filled with MOPS-buffered saline solution containing 1\% BSA. The vessels were handled according to the method described by Lindauer et al. [18]. In brief, the proximal end of the vessel segment was mounted on a glass micropipette and secured. The lumen was gently flushed to remove remaining blood. Next, the distal end was cannulated with the second glass micropipette and fixed. At this point, the organ chamber was placed at the stage of inverted microscope (CKX41, Olympus, Germany) equipped with a video camera and a monitor. Extraluminal fluid was replaced with MOPS-buffered saline solution without BSA, slowly heated to $37^{\circ} \mathrm{C}$ (using water bath) and exchanged at a rate of $20 \mathrm{ml} / \mathrm{min}$ with the help of a peristaltic pump (Masterflex, Cole Parmer, USA). The pressure-servo system connected to the inlet of the MCA was set to maintain transmural pressure at $80 \mathrm{~mm} \mathrm{Hg}$. The vessel was continuously perfused at a slow rate of $100 \mu \mathrm{l} / \mathrm{min}$.

Eighty five vessels were used for this study.

\section{Experimental protocols}

After 60 min equilibration, the MCA developed spontaneous tone by reducing the initial diameter by at least $30 \%$. Next, reactivity of the MCA to an increase in the extracellular $\mathrm{K}^{+}$concentration was tested by adding $20 \mathrm{mM} \mathrm{KCl}$ extraluminally. Only vessels which dilated in response to $\mathrm{KCl}$ by more than $20 \%$ were further studied.

Acute hyponatremia was induced in the chamber for $60 \mathrm{~min}$ prior to the reactivity tests by replacing intra- and extravascular normonatremic MOPS-buffered saline containing $145 \mathrm{mM} \mathrm{Na}^{+}$with the one containing $121 \mathrm{mM} \mathrm{Na}^{+}$. The osmolality of the solutions was $300 \pm 3$ and $268 \pm 5 \mathrm{mOsm} / \mathrm{kg} \mathrm{H}_{2} \mathrm{O}$, respectively.

Before performing the functional tests of the MCAs, the stability of the diameter of pressurized and perfused vessels was tested in a pilot study. For that purpose, after initial stabilization, MCAs were left undisturbed in normonatremic ( $n=3$ vessels) or hyponatremic ( $n=3$ vessels) MOPS-buffered saline solution for $180 \mathrm{~min}$ (time required to perform the longest experiment). During this time, the diameter of the MCA did not change by more than $4 \%$ of the baseline, which was not statistically significant.

The responses of MCAs to increasing intravascular pressure, to rising concentrations of endothelium- and NO-dependent vasodilator acetylcholine (Ach, $10^{-6} \mathrm{M}, 10^{-5} \mathrm{M}$ and $10^{-4} \mathrm{M}$ ), to nonselective nitric oxide synthase inhibitor ( $N^{G}$-nitro-L-arginine methyl ester, L-NAME, $10^{-5} \mathrm{M}$ ) and nitric oxide donor (S-nitroso- $N$-acetylpenicillamine, SNAP, $10^{-5} \mathrm{M}$ ), were studied in separate groups of arteries in normo- and hyponatremia. In addition, L-NAME $\left(10^{-5} \mathrm{M}\right)$ was administered in normonatremia prior to Ach $\left(10^{-4} \mathrm{M}\right)$ to demonstrate that relaxation of the MCA in response to Ach depends solely on NO.

The measured parameter was the internal diameter of the artery.

In order to study the myogenic response of the MCA (constriction of the artery in response to increasing intravascular pressure), intraluminal pressure was slowly lowered from 80 to $20 \mathrm{~mm} \mathrm{Hg}$ and then slowly raised to $40 \mathrm{~mm} \mathrm{Hg}, 60 \mathrm{~mm} \mathrm{Hg}, 80 \mathrm{~mm}$ $\mathrm{Hg}$ and $100 \mathrm{~mm} \mathrm{Hg}$. At each pressure level, the artery was equilibrated for $10 \mathrm{~min}$, and the diameter was measured. After that, intravascular pressure was set again at $80 \mathrm{~mm} \mathrm{Hg}$ and the artery was allowed to equilibrate for $15 \mathrm{~min}$ in calcium-free (1 mM EGTA) MOPS-buffered saline solution. Subsequently, the vessel went through intravascular pressure changes according to the paradigm used prior to EGTA administration. This enabled calculation of the diameter of MCA in percentage of maximal dilation at each pressure level according to the formula: $D_{C a} / D_{O C a} \times 100$, where $D_{C a}$ and $D_{O C a}$ are diameters measured in the presence and absence of $\mathrm{Ca}^{2+}$, respectively. In this series, 8 by 8 vessels were studied in normo- and hyponatremia.

Acetylcholine was administered intraluminally in the following concentrations: $10^{-6} \mathrm{M}, 10^{-5} \mathrm{M}$ and $10^{-4} \mathrm{M}$ and the diameter of the MCA was measured 15 min later. Only one concentration was studied in each experiment in order to avoid tachyphylaxis. After completion of the Ach reactivity test, the MCA was exposed for $15 \mathrm{~min}$ to calcium-free (1 mM EGTA) MOPS-buffered saline solution in order to maximally dilate the vessel. Response of MCA to acetylcholine was expressed as a percentage of maximal dilation according to the formula: $\left(D_{\text {Ach }}-D_{\text {baseline }}\right) /\left(D_{\max }\right.$ - $\left.D_{\text {baseline }}\right) \times 100 \%$, where $D$ denotes the diameter of the vessel: after Ach $\left(D_{\text {Ach }}\right)$, at baseline ( $\left.D_{\text {baseline }}\right)$ and during maximal dilation $\left(D_{\max }\right)$, respectively. The response to each dose of Ach was tested in 7 by 7 vessels in normo- and hyponatremia, i.e. 42 vessels were used. The highest concentration of Ach $\left(10^{-4} \mathrm{M}\right)$ was administered also 30 min following pretreatment 
with L-NAME in normonatremia to determine participation of NO in the vasodilation of the MCA observed in response to Ach. In this group, 5 vessels were used.

The remaining compounds were administered extraluminally in the concentration of $10^{-5} \mathrm{M}$, and the diameter of the MCA was measured 30 minutes (L-NAME, $n=7$ vessels in normonatremia and 5 vessels in hyponatremia) or 15 minutes (SNAP, 5 by 5 vessels in normo- and hyponatremia) later. Changes in the MCA diameter in both cases were expressed as a percentage of baseline.

Eighty five vessels were used for this study.

\section{Solutions and reagents}

MOPS-buffered saline solution contained (in $\mathrm{mM}$ ): MOPS 2.0; $\mathrm{NaCl} 144.0$ or 120.0 (for normo- and hyponatremia, respectively); $\mathrm{KCl} 3.0 ; \mathrm{CaCl}_{2} 2.5 ; \mathrm{MgSO}_{4}$ 1.5; pyruvate 2.0; glucose 5.0; EDTA 0.02; $\mathrm{NaH}_{2} \mathrm{PO}_{4} 1.21$.

MOPS-buffered saline solution was freshly prepared before the experiment. The tested compounds were dissolved directly in MOPS-buffered saline solution.

All compounds used in the study were purchased from Sigma-Aldrich.

\section{Statistical analysis}

Values are expressed as means \pm standard error of the mean (S.E.M.). Data were analyzed using ana-

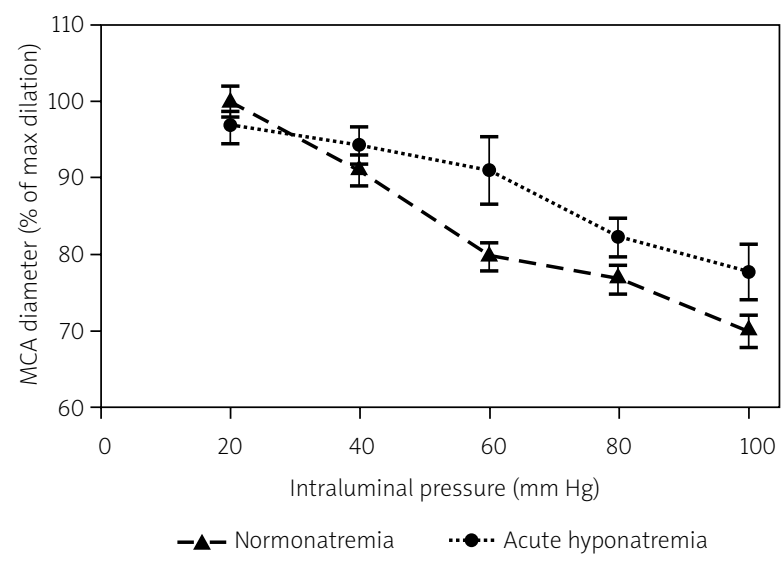

Fig. 1. Myogenic response of the middle cerebral artery (MCA) in normo- (filled triangles) and hyponatremia (filled circles). Diameter of the MCA is expressed as a percentage of the maximal dilation measured at each pressure in $\mathrm{Ca}^{2+}$-free solution. Data are presented as means \pm S.E.M., $n=7$ vessels in each groups. lysis of variance (ANOVA), followed by Tukey's multiple comparison tests, or paired or unpaired Student's $t$-test, as appropriate (Statistica 10). The $P$ values $<0.05$ were considered statistically significant.

\section{Results}

\section{Myogenic response}

Similar pressure-dependent constriction of the MCA was observed both in normonatremia and acute hyponatremia (Fig. 1). In both cases, in normal conditions (presence of $\mathrm{Ca}^{2+}$ in MOPS - buffered solution), the vessels constricted along with increasing intravascular pressure whereas in $\mathrm{Ca}^{2+}$-free conditions, vessels dilated when transmural pressure was raised. The ratio of the diameters recorded in the presence and absence of $\mathrm{Ca}^{2+}$ depicted in Fig. 1 in a function of time indicates that myogenic reactivity is preserved in acute hyponatremia.

\section{Responses to acetylcholine}

Intravascular administration of acetylcholine $\left(10^{-6} \mathrm{M}\right.$, $10^{-5} \mathrm{M}, 10^{-4} \mathrm{M}$ ) during normonatremia produced relaxation of MCA in a dose-dependent manner (Fig. 2) with the maximal effect observed at a concentration of $10^{-4} \mathrm{M}$

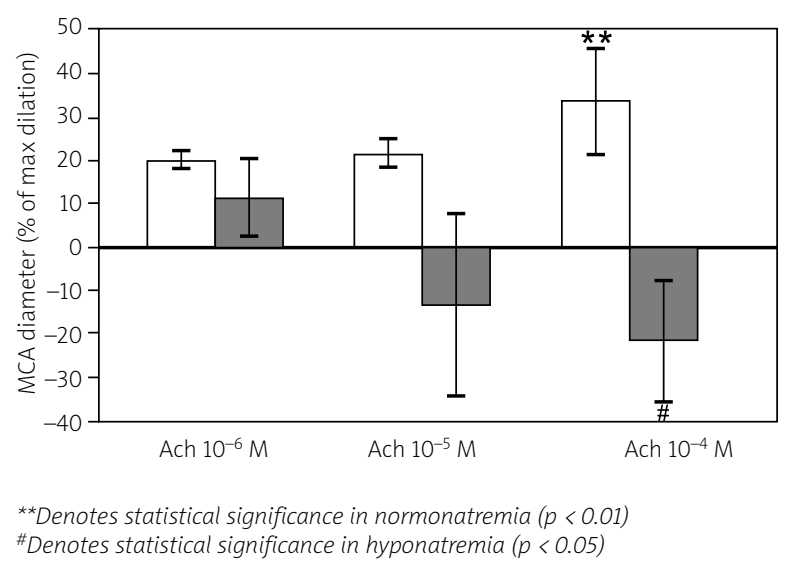

Fig. 2. The rat middle cerebral artery (MCA) dilates progressively in response to intravascular administration of increasing concentrations of acetylcholine (Ach) during normonatremia (white bars) but constricts during hyponatremia (gray bars). Diameter of the MCA is expressed as a percentage of the maximal dilation measured in $\mathrm{Ca}^{2+}$-free solution. Data are presented as means \pm S.E.M., $n=7$ vessels per each concentration of Ach in each group. 
(34 $\pm 12 \%, p<0.01)$. During hyponatremia, the increasing concentration of Ach resulted in a progressive contraction of the artery, which was statistically significant at a concentration of $10^{-4} \mathrm{M}(21 \pm 10 \%, p<0.05)$.

Pretreatment of the MCA in normonatremia with NO synthase inhibitor - L-NAME $\left(10^{-5} \mathrm{M}\right)$ eliminated dilation of this artery to $10^{-4} \mathrm{M}$ Ach indicating that relaxation of the MCA in response to Ach is entirely NO-dependent. In this group, 15 min after administration of Ach, MCA dilated by $39 \pm 12 \%(p<0.01)$ in the presence of NO (without L-NAME) but constricted by $14 \pm 4 \%(p<0.05)$ in the absence of NO (with L-NAME). The constriction of the MCA after administration of $10^{-4} \mathrm{M}$ Ach in this experiment did not statistically differ from the MCA response to $10^{-4} \mathrm{M}$ Ach in hyponatremia.

\section{Effect of NO synthase inhibition and NO supplementation}

Administration of L-NAME $\left(10^{-5} \mathrm{M}\right)$ led to a comparable constriction of the MCA in normonatremia $(27 \pm 3 \%$ of baseline, $p<0.05)$ and in hyponatremia ( $26 \pm 5 \%$ of baseline, $p<0.05$ ).

In order to explain further the impaired MCA response to NO- and endothelium-dependent Ach in hyponatremia, the endothelium-independent NO-donor SNAP $\left(10^{-5} \mathrm{M}\right)$ was administered extraluminally. The compound resulted in no change of the MCA diameter in hyponatremia, although it led to statistically significant dilation of the artery (by $43 \pm 5 \%$ of baseline, $p<0.001$ ) in normonatremia (Fig. 3).

\section{Discussion}

To our knowledge, this is the first study, which demonstrates that the regulation of the middle cerebral artery is impaired in moderate, acute hyponatremia.

The middle cerebral artery was chosen for this study, because being a resistance vessel it participates in the regulation of cerebral blood flow [7]. The important point is also that using isolated vessel enables eliminating parenchymal influences. Slow perfusion of the vessel and pressurization to the physiological level of blood pressure simulates in vivo conditions, ensures proper myogenic tone and shear stress. The latter is required to maintain tonic influence of endothelium on the vessel diameter [3]. The next advantage is the possibility to induce hyponatremia without the participation of hormones.

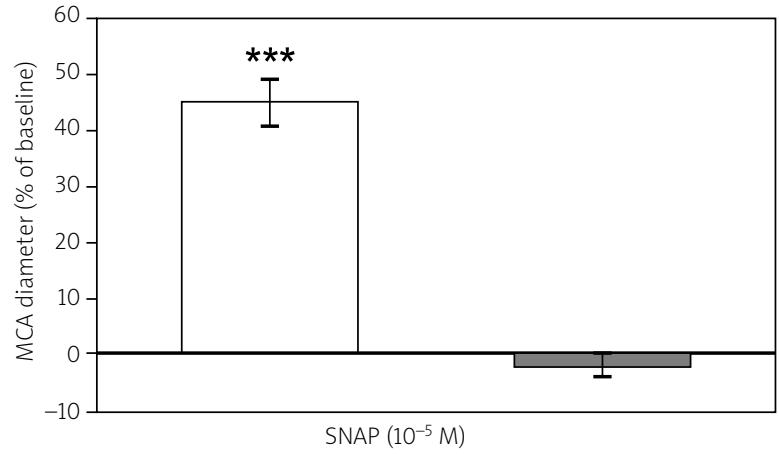

${ }^{* * *}$ Denotes statistical significance in comparison with the baseline diameter in normonatremia $(p<0.001)$

Fig. 3. The middle cerebral artery (MCA) dilates in response to the administration of nitric oxide donor (SNAP, $n=5$ in each group) in normonatremia (white bars) but does not dilate in hyponatremia (gray bars). Data are presented as means \pm S.E.M.

The main hormones involved in the regulation of water-electrolyte balance - antidiuretic hormone and natriuretic peptide, one of which is used to induce experimental hyponatremia in vivo $[15,33]$ are vasoactive [30,37]. Their presence would complicate the interpretation of the data.

Our experiments were designed to study the influence of acute, moderate hyponatremia on basic, albeit important aspects of vascular regulation.

Myogenic response of the MCA is essential for the maintenance of basal vascular tone as well as autoregulation of blood flow and capillary hydrostatic pressure [7]. This response, characterized by a decrease in vessel diameter when transmural pressure increases and by an increase in vessel diameter when transmural pressure decreases, has been studied in various size resistance cerebral blood vessels of different species including the rat MCA $[6,10,19]$. The response is related to the specific characteristic features of vascular smooth muscle cells (VSMC) and depends on the activation of voltage-dependent calcium channels. According to McCarron et al. [19], stretch-evoked enhancement of $\mathrm{Ca}^{2+}$ currents may also play a role. The counteracting ionic mechanism has also been postulated. According to the published data, the myogenic response is opposed by the activation of VSMC voltage- and $\mathrm{Ca}^{2+}$-dependent $\mathrm{K}^{+}$channels [1,5]. Therefore, if these $\mathrm{K}^{+}$channels are inhibited in VSMC in hyponatremia, as has been 
shown by $\mathrm{Wu}$ et al. [38], the myogenic response should be reinforced. This is not the case in our study, which shows that MCA constricts to a similar degree in response to the increase in intraluminal pressure both in normo- and hyponatremia. Lack of the effect of acute moderate hyponatremia on the myogenic response was also reported by Wang et al. [34] in renal afferent arteriole.

Besides showing normal myogenic response of the MCA in hyponatremia, our study indirectly suggests that constriction ability of VSMC is not affected by hyponatremia. This suggestion arises from the observation that the degree of contraction of the MCA in response to Ach is comparable in hyponatremia and normonatremia with NO synthesis inhibited. When NO is not available, Ach acts directly on vascular smooth muscle cells which results in contraction.

Also, shear stress-related inhibition of the tone of the MCA by NO seems to be unchanged in hyponatremia. Inhibition of the synthesis of NO in the present study resulted in a similar degree of vasoconstriction in normo- and hyponatremia. One can argue that L-NAME - the inhibitor which we have used to eliminate constitutive production of NO is not a selective one and results in the inhibition of both endothelial and neuronal isoforms of NOS. Thus, the constriction of the MCA in response to L-NAME in our study could partially result from the elimination of $\mathrm{NO}$ released from nitrergic nerve endings in the wall of MCA [20]. What is more, the presence of functionally active nitrergic nerve endings in the isolated middle cerebral artery of the rat has been demonstrated [18]. Although elimination of NO-containing nerve endings from the MCA in the study of Lindauer et al. [18] resulted in only slight constriction of the MCA in the isotonic medium, the enhanced basal release of NO from those nerves in hyponatremia cannot be excluded. Particularly, in view of the data, demonstrating hypoosmolarity-induced release of neurotransmitters from rat brain cortical synaptosomes [31].

The interpretation of our data is also difficult because the effect of NO on the rat MCA is complex. In addition to the well-known mechanism of action connected with the activation of a soluble guanylyl cyclase and increased generation of cyclic guanosine monophosphate (cGMP), NO inhibits the synthesis of 20-hydroxyeicosatetraenoic acid (20-HETE) in VSMC [29]. The latter is a product of cytochrome
P450-dependent hydroxylation of arachidonic acid and an inhibitor of VSMC large conductance calciumdependent $\mathrm{K}^{+}$channels which were mentioned earlier among the channels activated during RVD [23]. According to Sun et al. [29], activation of cGMP and disinhibition of $\mathrm{BK}_{\mathrm{ca}}$ participate equally in the NO-dependent relaxation of the MCA of the rat.

The first finding, which clearly demonstrates the impaired regulation of the MCA in hyponatremia in our study is the loss of the dilation of the vessel in response to Ach. Physiologically, relaxation to Ach depends on the integrity of the endothelium and, in the rat MCA, is NO-dependent $[3,13]$. The latter is also confirmed in the present study by the constriction of the MCA in normonatremia when Ach is administered in the absence of NO. Therefore, the loss of the normal MCA response to Ach in hyponatremia may be considered as an impairment of agonist-induced eNOS activation [17]. On the other hand, lack of the dilation of the MCA in hyponatremia after administration of NO donor, which appeared to be a very powerful dilator in normonatremia, leads to the conclusion that impaired NO-dependent signal transduction in VSMC is the basis of the observed dysfunction.

While the exact mechanism behind impairment of NO-dependent signal transduction in hyponatremia remains to be determined, it is worth mentioning that NO is not only involved in the regulation of flow- and agonist-dependent relaxation [3] of cerebral vessels but also serves a permissive function in promoting dilation to other vasoactive factors $[4,11,35]$.

Therefore, disturbed NO-dependent signalling in vascular smooth muscle in hyponatremia may have an important impact on the regulation of cerebral blood flow.

Regarding the clinical importance of our results, dysfunction of NO-dependent regulation may impair a cerebrovascular response to metabolic acidosis, hypercapnia as well as to the increase in extracellular potassium concentration [11,35]. This may have particular consequences in the presence of underlying neurological diseases or non-neurological metabolic abnormalities.

In conclusion, our study shows that acute hyponatremia impairs some of the important mechanisms of the regulation of the middle cerebral artery, which may contribute, in addition to the osmotic brain swelling, to the disturbed intracranial homeostasis associated with hyponatremia. 


\section{Acknowledgements}

This study was supported by a grant of the Polish Ministry of Science and Higher Education [N401 19032/3924] and by the Mossakowski Medical Research Centre PAS.

The authors are grateful for the help with setting up the method of isolated vessel studies to Dr. Ute Lindauer and Mr. Marco Foddis from the Experimental Neurology at Charite Berlin, Dr. Robert Bryan Jr. and Dr. Sean Marrelli from Texas Baylor Institute for the valuable discussions of the method.

Authors report no conflict of interest.

\section{References}

1. Albarwani S, Nemetz LT, Madden JA, Tobin AA, England SK, Pratt PF, Rusch NJ. Voltage-gated K+ channels in rat small cerebral arteries: molecular identity of the functional channels. J Physiol 2003; 551: 751-763.

2. Anderson RJ, Chung H-M, Kluge R, Schrier RW. Hyponatremia: a prospective analysis of its epidemiology and the pathogenetic role of vasopressin. Ann Intern Med 1985; 102: 164-168.

3. Andresen J, Shafi NI, Bryan RM Jr. Endothelial influences on cerebrovascular tone. J Appl Physiol 2006; 100: 318-327.

4. Barkoudah E, Jaggar JH, Leffler CW. The permissive role of endothelial NO in CO-induced cerebrovascular dilation. Am J Physiol Heart Circ Physiol 2004; 287: H1459-H1465.

5. Brayden J, Nelson M. Regulation of arterial tone by activation of calcium-dependent potassium channels. Science 1992; 256: 532-535.

6. Coulson RJ, Chesler NC, Vitulio L, Cipolla MJ. Effects of ischemia and myogenic activity on active and passive mechanical properties of rat cerebral arteries. Am J Physiol Heart Circ Physiol 2002; 283: H2268-H2275.

7. Faraci FM, Heistad DD. Regulation of large cerebral arteries and cerebral microvascular pressure. Circ Res 1990; 66: 8-17.

8. Fraser JF, Stieg PE. Hyponatremia in the neurosurgical patient: epidemiology, pathophysiology, diagnosis, and management. Neurosurg 2006; 59: 222-229.

9. Furchgott, RF, Zawadzki, JV. The obligatory role of endothelial cells in the relaxation of arterial smooth muscle by acetylcholine. Nature (London) 1980; 288: 373-376.

10. Golding EM, Robertson TS, Bryan RM Jr. Comparison of the myogenic response in rat cerebral arteries of different calibers. Bran Res 1998; 785: 293-298.

11. Golding EM, Steenberg ML, Johnson TD, Bryan RM Jr. Nitric oxide in the potassium-induced response of the rat middle cerebral artery: a possible permissive role. Bran Res 2001; 889: 293-298.

13. Iadecola C, Pellegrino DA, Moskowitz MA, Lassen NA. Nitric oxide synthase inhibition and cerebrovascular regulation. J Cereb Blood Flow Metab 1994; 14: 175-192.

14. Kimura C, Koyama T, Oike M, Ito Y. Hypotonic stress-induced NO production in endothelium depends on endogenous ATP. Biochem Biophys Res Commun 2000; 274: 736-740.
15. Kozniewska E, Roberts TP, Vexler ZS, Oseka M, Kucharczyk J, Arieff Al. Hormonal dependence of the effects of metabolic encephalopathy on cerebral perfusion and oxygen utilization in the rat. Circ Res 1995; 76: 551-558.

16. Kitazono T, Faraci FM, Taguchi H, Heistad DD. Role of potassium channels in cerebral blood vessels. Stroke 1995; 26: 1713-1723.

17. Khurana VG, Feterik K, Springett MJ, Eguchi D, Shah V, Katusic ZS. Functional interdependence and colocalisation of endothelial nitric oxide synthase and heat shock protein 90 in cerebral arteries. J Cereb Blood Flow Metab 2000; 20: 1563-1570.

18. Lindauer U, Kunz A, Schuh-Hofer S, Vogt J, Deier JP, Dirnagl U. Nitric oxide from perivascular nerves modulates cerebral arterial pH reactivity. Am J Physiol Heart Circ Physiol 2001; 281: $1353-$ 1363.

19. McCarron JG, Crichton CA, Langton PD, MacKenzie A, Smith GL. Myogenic contraction by modulation of voltage-dependent calcium currents in isolated rat cerebral arteries. J Physiol 1997; 498: 371-379.

20. Okamura T, Ayajiki K, Fujioka H, Shinozaki K, Toda N. Neurogenic cerebral vasodilation mediated by nitric oxide. Jpn J Pharmacol 2002; 88: 32-38.

21. Palmer BF. Hyponatremia in patient with central nervous system disease: SIADH versus CSW. Trends Endocr Metab 2003; 14: 182-187.

22. Perry PB, O'Neill WC. Swelling-activated $K$ fluxes in vascular endothelial cells: volume regulation via $\mathrm{K}-\mathrm{Cl}$ cotransport and K channels. Am J Physiol 1993; 265 (3 Pt 1): C763-769.

23. Pasantes-Morales H, Morales Mulia S. Influence of calcium on regulatory volume decrease: role of potassium channels. Nephron 2000; 86: 414-427.

24. Pasantes-Morales H, Lezama RA, Ramos-Mandujano G, Tuz KL. Mechanisms of cell volume regulation in hypo-osmolality. Am J Med 2006; 119: S4-S11.

25. Rosner MH. Exercise-associated hyponatremia. Semin Nephrol 2009; 29: 271-281.

26. Schettini A, Stahurski B, Young HF. Osmotic and osmotic-loop diuresis in brain surgery. Effects on plasma and CSF electrolytes and ion excretion. J Neurosurg 1982; 56: 679-684.

27. Spino M, Sellers EM, Kaplan HL, Stapleton C, MacLeod SM. Adverse biochemical and clinical consequences of furosemide. Can Med Assoc J 1978; 118: 1513-1518.

28. Spormann R, Harding U, Stuhr M, Röther J, Puschel U, Reifferscheid F. Serious complications following a marathon run: interactive case report of a dramatic course. Anaesthesiol Intensivmed Notfallmed Schmerzther 2012; 47: 696-702.

29. Sun C-W, Falck JR, Okamoto H, Harder DR, Roman RJ. Role of CGMP versus 20-HETE in the vasodilator response to nitric oxide in rat cerebral arteries. Am J Physiol Circ Physiol 2000; 279: H339-H350.

30. Takayasu M, Kajita Y, Suzuki Y, Shibuya M, Sugita K, Ishikawa T, Hidaka $H$. Triphasic response of rat intracerebral arterioles to increasing concentration of vasopressin in vitro. J Cereb Blood Flow Metab 1993; 13: 304-309.

31. Tuz K, Peña-Segura C, Franco R, Pasantes-Morales H. Depolarization, exocytosis and amino acid release evoked by hyposmolarity from cortical synaptosomes. Eur J Neurosci 2004; 19: 916-924. 
32 Upadhyay A, Jaber BL, Madias NE. Incidence and prevalence of hyponatrermia. Am J Med 2006; 119: S30-S35.

33. Verbalis JG. Hyponatremia induced by vasopressin or desmopressin in female and male rats. Am J Soc Nephrol 1993; 3: 1600-1606.

34. Wang X, Takeya K, Aaronson PI, Loutzenhiser K, Loutzenhiser R. Effects of amiloride, benzamil, and alterations in extracellular $\mathrm{Na}^{+}$on the rat afferent arteriole and its myogenic response. Am J Physiol Renal Physiol 2008; 295: F272-F282.

35. Wang Q, Bryan RM, Pelligrino DA. Calcium-dependent and ATP-sensitive potassium channel and the permissive function of cyclic GMP in hypercapnia-induced pial arteriolar relaxation. Brain Res 1998; 793: 187-196.

36. Welsh DG, Nelson MT, Eckman DM, Brayden JE. Swelling-activated cation channels mediate depolarization of rat cerebrovascular smooth muscle by hypoosmolarity and intravascular pressure. J Physiol 2000; 527: 139-148.

37. Woodard GE, Rosado JA. Natriuretic peptides in vascular phys iology and pathology. Int Rev Cell Mol Biol 2008; 268: 59-93.

38. Wu B-N, Luykenaar KD, Brayden JE, Giles WR, Corteling RL, Wiehler WB, Welsh DG. Hyposmotic challenge inhibits inward rectifying K+ channels in cerebral arterial smooth muscle cells. Am J Physiol Circ Physiol 2007; 292: H1085-H1094. 